\title{
A 14.8 Mb 12p Deletion Disrupting ETV6 in a Patient with Myelodysplastic Syndrome
}

Angelo Valetto ${ }^{1 *}$, Veronica Bertini ${ }^{1}$, Elena Ciabatti ${ }^{2,3}$, Maria Immacolata Ferreri ${ }^{1}$, Alice Guazzelli ${ }^{1}$, Antonio Azzarà ${ }^{2}$, Susanna Grassi ${ }^{2}$, Alessia Azzarà ${ }^{1}$, Francesca Guerrini ${ }^{2}$, lacopo Petrini ${ }^{2,4}$ and Sara Galimberti ${ }^{2}$

${ }^{1}$ Laboratory of Medical Genetics, A.O.U.P., S. Chiara Hospital, Pisa, Italy

${ }^{2}$ Department of Clinical and Experimental Medicine, Section of Hematology, University of Pisa, Pisa, Italy

${ }^{3} \mathrm{GenOMeC}$, University of Siena

${ }^{4}$ Department of translational Research and New Technologies in Medicine, University of Pisa, Pisa, Italy

*Corresponding author: Valetto A, Laboratory of Medical Genetics, A.O.U.P., S. Chiara Hospital, Pisa, Italy, Tel: +39050992777; E-mail: a.valetto@ao-pisa.toscana.it

Received Date: Oct 27, 2016; Accepted Date: Mar 16, 2017; Published Date: Mar 21, 2017

Copyright: (C) 2017 Valetto A, et al. This is an open-access article distributed under the terms of the Creative Commons Attribution License, which permits unrestricted use, distribution, and reproduction in any medium, provided the original author and source are credited.

\begin{abstract}
We present on a new case of myelodysplastic syndrome characterized by array Comparative Genomic Hybridization. This technique confirmed the monosomy 7 , detected by conventional cytogenetics, and revealed also a deletion on the short arm of chromosome 12. This deletion extends for about $14.8 \mathrm{Mb}$ and breaks ETV6 gene.

$12 p$ deletion extents in hematological malignancies may vary, but the minimally deleted region almost invariably contains ETV6, that is considered the main candidate tumor suppressor genes within the region for tumor progression. It has been shown that levels of ETV6 were significantly decreased in cases with $12 \mathrm{p} 13$ deletions, whereas expression of other genes in the deleted region, like BCL2L14, LRP6, DUSP16 and GPRC5D, did not show any variation, independently of their copy number status. This observation strengthens the fact that ETV6 may play a potential role in the tumorigenesis process. The role of ETV6 in our patient myelodysplastic syndrome is showed by his clinical history and his poor prognosis.
\end{abstract}

Keywords: Myelodysplastic syndrome; 12p Deletion; CGH array; ETV6

\section{Introduction}

Myelodysplastic syndromes (MDS) are a heterogeneous group of clonal hematopoietic stem cell malignancies characterized by peripheral blood cytopenias, one or more lineage dysplasia, and ineffective hematopoiesis. Metaphase cytogenetics has become the routine test in the management of MDS as well as in hematological malignancies where the presence of specific chromosomal aberrations can be diagnostic, or highly predictive of prognosis or responsiveness to target therapeutics. Clonal cytogenetics abnormalities have been observed in about $50 \%$ of de novo MDS and in more than $80 \%$ of secondary MDS cases [1-4].

Karyotype analysis has technical limitations, mainly due to the available metaphase spreads and to the chromosome resolution. These factors result in the underestimation of the degree of chromosomal changes. In the recent years, the advent of array CGH (Comparative Genomic Hybridization) has given a great contribution to the diagnostic tools available for MDS, overcoming some of the limitations of the classic cytogenetic techniques. This method do not rely on cell division, has superb resolution for unbalanced lesion and allows for detection of copy number variation that can be related to the tumor progression $[1,5,6]$.

Here, we present a case of MDS where we were able to find a chromosome 12 abnormality detected only by array CGH.

\section{Material and Methods}

\section{Patient history}

In October 2005, a 60-year-old male patient was admitted to our hospital for nodal marginal zone non-Hodgkin's lymphoma, stage IV (bone marrow was infiltrated), diagnosed after biopsy of a $20-\mathrm{mm}$ inguinal lymph-node. At that time, conventional cytogenetics on bone marrow was normal. Patient was firstly treated with 2-chlorodeoxiadenosine for 6 cycles and subsequently, because of a poor response, according to R-CHOP (rituximab, cyclophosphamide, vincristine, prednisone) schedule. After further three months, our patient showed again enlarged lymph-nodes in abdomen and neck, thus he received R-GDP (rituximab, gemcitabine, cisplatin, and dexamethasone). In February 2008, because of a relapse in the central nervous system (CNS), he received high-dose methotrexate, intratecal aracytin and temozolomide. This latest drug was repeated in February 2011 for a new evidence of a further disease progression in CNS, this time followed also by encephalic radiotherapy. In August 2011, for newly enlarged lymph-nodes in abdomen, he received $\mathrm{R}$ bendamustine, achieving a partial remission, without significant hematological or extra-hematological toxicities. Nevertheless, in November 2012, he presented with anemia (hemoglobin $=9.7 \mathrm{~g} / \mathrm{dL}$ ), thrombocytopenia (platelets $=25 \times 10^{9} / \mathrm{L}$ ) and leukocytosis with monocytosis (monocytes $=2.8 \times 10^{9} / \mathrm{L}$ ).

The bone marrow showed an increased cellularity, with $25 \%$ of monocytic precursors, $7 \%$ of CD34+, CD11b-, CD13+ blasts, reduced erythroid and megakaryocytic lineage, with clear dysplastic features. 
Patient was then diagnosed as affected by chronic myelomonocytic leukemia type 1 .

Patient received epoietin and danazol, but he rapidly became transfusion-dependent. On this bases, the CPSS risk score of our patient was high [7].

For a further increase of leukocytes, patient received etoposide for three months, but he died in November 2013 because of sepsis.

\section{Genetic analysis}

Standard karyotype was performed on metaphase spreads obtained by unstimulated cultures of BM blood sample.

Array CGH was performed according to standard protocols. Genomic DNA was extracted from $200 \mu \mathrm{L}$ of bone marrow anticoagulated by EDTA. This DNA and a male reference DNA were labeled differentially with Cyanine 3 and Cyanine 5, using standard procedures. Microarray experiments were performed on $4 \mathrm{x} 44 \mathrm{~K}$ microarrays (Agilent Technologies, Santa Clara, CA), that have $\sim 35 \mathrm{~kb}$ average probe spatial resolution. Microarrays were scanned with an Agilent scanner G256BA. Hybridization data analysis was carried out with Agilent Technologies dedicated softwares. Search for Copy Number Variation Regions (CNVRs) was performed using the Database of Genomic Variants (http://projects.tcag.ca/variation/).

WT1 gene expression and mutations of ASXL1, TP53, EZH2, and TET2 genes were evaluated according to standard procedures. DNA was extracted from the bone marrow samples by the QIAamp DNA Blood Mini Kit (Qiagen, Hilden, Germany). About 600 ng of DNA were analysed for each patients by using a custom qBiomarker somatic mutation PCR array (Qiagen, Hilden, Germany) for ASXL1, EZH2, TET2, and TP53 gene. This method can detect as low as $1 \%$ somatic mutations in the background of wild-type genomic DNA. Allele specific amplification is achieved by Amplification Refractory Mutation System (ARMS) technology, which is based on the discrimination by Taq polymerase between a match and a mismatch at the 3' end of the PCR primer. The analysis of data was performed using a pool of five healthy donors as control and calculating the relative abundance of mutant DNA templates by the formula $\Delta \mathrm{Ct}$ sample $=(\mathrm{Ct}$ mut $-\mathrm{Ct}$ ref) $<\Delta \mathrm{Ct}$ controls where the reference assay is designed on a non-variable region of the same gene which carries the mutation.

\section{Results}

Standard cytogenetic analysis detected a monosomy of chromosome 7 in about $70 \%$ of the metaphases. WT1 gene expression was in the normal range, and we did not find any mutations of $A S X L 1, T P 53$, $E Z H 2$, and TET2 genes.

Array CGH confirmed the monosomy of chromosome 7 (mean fluorescent ratio -0.66 ) and detected a chromosome $12 p$ interstitial deletion, from 12p13.2 to 12 p11.23 (mean fluorescent ratio -0.68), not visible at the karyotype analyzed by two expert cytogenetists. The deletion extends for about $14.8 \mathrm{Mb}$, starting from $11,897,416$ to $26,698,489 \mathrm{bp}$, and contains more than 55 annotated genes, according to UCSC database (http://genome.ucsc.edu/). Interestingly, the 12p13.2 breakpoint falls into ETV6 gene, which results partially deleted.

The karyotype was given as $\operatorname{arr}[$ GRCh37]
7p22.3q36.3 (92532_158909679)
12p13.2p11.23 $\left(11897416 \_26698489\right) \times 1[0.7]$ according to ISCN 2016
nomenclature.

\section{Discussion}

$12 p$ deletions are detected in a broad spectrum of hematological malignancies in acute myeloid leukemia (AML) they are usually associated with complex karyotypes, whereas in myelodysplastic syndromes these anomalies are often seen along with monosomy 7 [8-11]. Rucker et al. showed that about half of the AML with complex karyotype (CK-AML) presented $12 \mathrm{p} 13$ deletions, when analyzed by high-resolution arrays [12]. Moreover, in a series of 125 patients with secondary MDS, unbalanced translocations involving chromosome $12 \mathrm{p}$ were found in $8 \%$ of cases [4].

Deletion extents may vary, but the minimally deleted region almost invariably contains ETV6, that is considered the main candidate tumor suppressor genes within the region for tumor progression $[13,14]$. Other genes within the minimal deleted region like $C D K N 1 B$, $B C L 2 L 14, L R P 6, D U S P 16$ and GPRC5D may play an additional role in tumorigenesis and leukemogenesis [11]. All these genes are deleted in the case here presented; his deletion extends to band p11.23 where the oncogene KRAS2 is harbored.

ETV6 (ets translocation variant gene 6) is a member of the ETS transcription factor family, and putative tumor suppressor gene since it induces G1 arrest and blocks Ras-induced transformation, promotes apoptosis, and activates TP53 dependent pathways [13].

Alterations of ETV6, as a consequence of translocations, deletions, or somatic mutations, has been detected in myeloid malignancies and MDS with highly variable frequency; this variability mainly depends on the characteristics of the study population and on the technique used. Anyway, it can be assumed that at least 5\% of MDS/AML have ETV6 deletion $[15,16]$. This frequency has been shown to be much higher in association with monosomy $7[12,17,18]$. Wall et al. tested ETV6 deletion in a series of 38 patients with diagnosis of MDS/MPN (myeloproliferative neoplasms) or AML with monosomy 7 [19]. 6 out of 38 patients (16\%) showed an ETV6 deletion, that, in 5/6 was undetectable by conventional cytogenetic methods, as observed in our case. The breakpoints were heterogeneous: deletions could be complete, involving the entire ETV6 gene, or only partial, with the 5' of the gene retained. As it can be seen in Figure 1, in our patient ETV6 deletion is partial: the probes till to $11,847,532 \mathrm{bp}$ resulted not deleted, whereas the probes starting from $11,897,616 \mathrm{bp}$ indicates a monoallelic deletion (Figure 1).

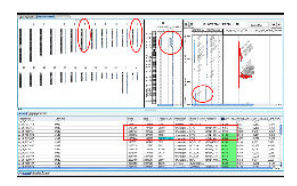

Figure 1: Array CGH profile. Array CGH profile showing all the chromosomes, with chromosome 7 partial monosomy and chromosome 12p deletion highlighted (top left, red circles). Profile of chromosome 12 is enlarged, showing the $12 \mathrm{p}$ deletion in detail (top right). ETV6 gene is partially deleted, as shown by the fluorescent ratios of the probes covering this gene (bottom).

The mean fluorescent ratio of the deletion $(-0.66)$ is compatible with a mosaic deletion, roughly involving about the $75 \%$ of the cells. This percentage is overlapping with the one with monosomy 7 , detected both by standard karyotype and array CGH. 
It can be guessed that this partial deletion results in a decreased expression of this gene [11]. These authors demonstrated that levels of ETV6 were significantly decreased in cases with 12 p13 deletions encompassing the entire gene or at least the $3^{\prime} \mathrm{UTR}$, like in the present case, whereas expression of other genes in the deleted region, like $B C L 2 L 14, L R P 6, D U S P 16$ and GPRC5D, did not show any variation, independently of their copy number status. This observation highlights that expression of ETV6 is strictly regulated in the blood cells and suggests a potential role for this gene in the tumorigenesis process. The role of ETV6 in the clinical history of our patient is showed by the poor prognosis both of lymphoma and of MDS.

\section{Conclusion}

Array CGH is a new diagnostic test to be performed along with conventional karyotype in MDS, especially when chromosome quality is poor. Discovering new cases with 12 p deletions, often undetectable by conventional karyotype, can be useful to establish if these deletions may identify a MDS subset with a negative prognostic factor.

Future studies investigating clonal evolution should clarify whether haploinsufficiency of ETV6 may play an early role in the process of leukemic transformation by disordering key processes of differentiation and proliferation and whether it also plays a critical role in the induction of chromosomal instability finally resulting in the development of -7 clones and/or clones with CK.

\section{References}

1. Makishima H, Rataul M, Gondek LP, Huh J, Cook JR, et al. (2010) FISH and SNP-A karyotyping in myelodysplastic syndromes: improving cytogenetic detection of del(5q), monosomy $7, \operatorname{del}(7 q)$, trisomy 8 and del(20q). Leuk Res. 34: 447-453.

2. Kawankar N, Vundinti BR (2011) Cytogenetic abnormalities in myelodysplastic syndrome: an overview. Hematology. 16: 131-138.

3. Giagounidis A, Haase D (2013) Morphology, cytogenetics and classification of MDS. Best Pract Res Clin Haematol. 26: 337-353.

4. Jhanwar SC (2014) Genetic and epigenetic pathways in myelodysplastic syndromes: A brief overview. Adv Biol Regul 58: 28-37.

5. Steensma DP, List AF (2005) Genetic testing in the myelodysplastic syndromes: Molecular insights into hematologic diversity. Mayo Clin Proc. 80: 681-698.

6. Thiel A, Beier M, Ingenhag D, Servan K, Hein M, et al. (2011) Comprehensive array CGH of normal karyotype myelodysplastic syndromes reveals hidden recurrent and individual genomic copy number alterations with prognostic relevance. Leukemia 25: 387-399.

7. Such E, Germing U, Malcovati L, Cervera J, Kuendgen A, et al. (2013) Development and validation of a prognostic scoring system for patients with chronic myelomonocytic leukemia. Blood 121: 3005-3015.
8. Streubel B, Sauerland C, Heil G, Freund M, Bartels H, et al. (1998) Correlation of cytogenetic, molecular cytogenetic, and clinical findings in 59 patients with ANLL or MDS and abnormalities of the short arm of chromosome 12. Br J Haematol 100: 521-533.

9. Grimwade D, Hills RK, Moorman AV, Walker H, Chatters S, et al. (2010) Refinement of cytogenetic classification in acute myeloid leukemia: determination of prognostic significance of rare recurring chromosomal abnormalities among 5876 younger adult patients treated in the United Kingdom Medical Research Council trials. Blood 116: 354-365.

10. Schanz J, Tüchler H, Solé F, Mallo M, Luño E, et al. (2012) New comprehensive cytogenetic scoring system for primary myelodysplastic syndromes (MDS) and oligoblastic acute myeloid leukemia after MDS derived from an international database merge. J Clin Oncol 30: 820-829.

11. Feurstein S, Rücker FG, Bullinger L, Hofmann W, Manukjan G, et al. (2014) Haploinsufficiency of ETV6 and CDKN1B in patients with acute myeloid leukemia and complex karyotype. BMC Genomics 15: 784.

12. Rücker FG, Bullinger L, Schwaenen C, Lipka DB, Wessendorf S, et al. (2006) Disclosure of candidate genes in acute myeloid leukemia with complex karyotypes using microarray-based molecular characterization. J Clin Oncol 24: 3887-3894.

13. De Braekeleer E, Douet-Guilbert N, Morel F, Le Bris MJ, Basinko A, et al. (2012) ETV6 fusion genes in hematological malignancies: a review. Leuk Res 36: 945-961.

14. Padron E, Yoder S, Kunigal S, Mesa T, Teer JK, al. (2014) ETV6 and signaling gene mutations are associated with secondary transformation of myelodysplastic syndromes to chronic myelomonocytic leukemia. Blood 123: 3675-3677.

15. Suela J, Alvarez S, Cigudosa JC (2007) DNA profiling by arrayCGH in acute myeloid leukemia and myelodysplastic syndromes. Cytogenet Genome Res 118: 304-309.

16. Tiu RV, Gondek LP, O'Keefe CL, Huh J, Sekeres MA, et al. (2009) New lesions detected by single nucleotide polymorphism array-based chromosomal analysis have important clinical impact in acute myeloid leukemia. J Clin Oncol 27: 5219-5226.

17. Heinrichs S, Kulkarni RV, Bueso-Ramos CE, Levine RL, Loh ML, et al. (2009) Accurate detection of uniparental disomy and microdeletions by SNP array analysis in myelodysplastic syndromes with normal cytogenetics. Leukemia. 23: 1605-1613.

18. Kolquist KA, Schultz RA, Furrow A, Brown TC, Han JY, et al. (2011) Microarray-based comparative genomic hybridization of cancer targets reveals novel, recurrent genetic aberrations in the myelodysplastic syndromes. Cancer Genet 204: 603-628.

19. Wall M, Rayeroux KC, MacKinnon RN, Zordan A, Campbell LJ (2012) ETV6 deletion is a common additional abnormality in patients with myelodysplastic syndromes or acute myeloid leukemia and monosomy 7 . Haematologica 97: 1933-1936. 